\title{
Effectiveness of Manual therapy followed by Active Exercises to improve the Functional Ability of Women with Neck Pain
}

\author{
Heba Haider ${ }^{1 *}$, Fakiha Farooqui ${ }^{2}$, Vinod Kumar ${ }^{3}$, Subbayal Shakeel ${ }^{4}$, M Faizan Hassan ${ }^{5}$, Sehar Pasha ${ }^{6}$, Saba \\ Sabir ${ }^{7}$ and Adiba $^{8}$
}

${ }^{1}$ Physical Therapist, Mamji Hospital

${ }^{2}$ Physical Therapist, Baqai Hospital

${ }^{3}$ Assistant Professor, Isra Institute of Rehabilitation Sciences, Isra University, Karachi Campus

${ }^{4}$ Senior Lecturer, Bhitai Institute of Physiotherapy and Rehabilitation Sciences, Mirpurkhas

${ }^{5}$ Physical Therapist, Ziauddin University

${ }^{6}$ Senior Lecturer, Isra Instituite of Rehabilitation Sciences, Isra University

${ }^{7}$ Physical Therapist, Buch Physio Care, Multan

${ }^{8}$ Physical Therapist, Shaheed Mohtarma Benazir Bhutto Institute of trauma.

Submission: August 21, 2019; Published: September 03, 2019

*Corresponding author: Heba Haider, Physical Therapist, Mamji Hospital

Abstract

Objective: The purpose of this study was to observe the effectiveness of manual therapy followed by active exercises to improve the functional ability of working and non-working women with neck pain. The results of this study would indicate to physiotherapist about treatment plan, used for the treatment of neck pain that would potentially increase the cervical range of motion and / or decrease pain experienced by the patient, improving the functional ability to work for their activities of daily living and leading to a more effective treatment protocol. The outcome of the study will help physiotherapist to select more appropriate treatment for patients based on the outcomes and clinical findings.

Method: This was achieved by selecting 34 female subjects between the ages of 30 to 60 years were assessed through Muscle Length Test of Levator scapulae, Sternocleidomastoid, Upper Trapezius, Scalene (anterior, posterior, medius), Spurling's test, Distraction test, Vernon Neck Pain Disability Index and Numeric Pain Rating Scale and a reassessment was taken after follow-up of 2 weeks. Patient received treatment session, 6 days of physical therapy visits in a week in which first rib depression manipulation was given 3 sessions in a week followed by 3 sessions of active exercise program which included stretching and strengthening exercises. Patients were classified as having successful or unsuccessful outcomes.

Result: From a randomly selected patients of working and non-working, 34 female patients,20 (14 acute \& 6 chronic conditions which included 15 middle age and 5 old age women) patients received effective treatment and had NDI score less than 14\%, with reduce sign and symptoms and improved strength of cervical muscles with greater functional ability so they were send back to their work while 12 (12 chronic condition which included 6 old age and 6 middle age women) patients did not receive more effective treatment as their NDI score was more than $15 \%$ with aggravating sign and symptoms and reduced strength of cervical muscles with lesser functional ability. 2 (chronic old age) patients did not receive any improvement after the course of 2-week treatment sessions.

Conclusion: The implications of the findings and outcomes of this study shows that there is affectivity of first rib depression manipulation with exercise program but in acute conditions rather than chronic conditions and it is a suggestion to increase the time duration of treatment sessions for patients in chronic conditions which might reduce their sign and symptoms and neck pain for better functional ability or different techniques of manual therapy can also be used for better results which is further discussed.

Keywords: Neck Pain; Cervical Spine; Flexion; Extension; Right Lateral Flexion; Left Lateral Flexion; Pinched Nerve; Anxiety; Stress; Sleeping in Awkward Position; Poor Posture; Heavy Physical Work; Degeneration; Mobilization; Manipulation Techniques; Sub-Acute Neck Pain; Massage; Stimulate; Static Contraction

\section{Introduction}

Neck pain disorders remain a common problem in modern industrialized countries. Neck pain is a commonly reported problem that affects $70 \%$ of individual at sometimes in their lives. Prevalence is generally higher in women, whether there is a working or non-working woman, higher in high-income 
countries compared with low- and middle-income countries and higher in urban areas compared with rural areas. Many environmental and personal factors influence the onset and course of neck pain. A higher incidence of neck pain among women and an increased risk of developing neck pain until the 35-49-year age group, after which the risk begins to decline [1]. It can be caused by a number of factors, including muscle strain, ligament sprains, arthritis, or a «pinched» nerve, anxiety/ stress, sleeping in awkward position, poor posture, heavy physical work, degeneration etc. Mobilization and manipulation techniques are moderately effective in acute and sub-acute neck pain at short term and intermediate term follow up [2]. In the long term, exercises alone or combined with manual therapies were superior over manual therapies used alone [3].

In this respect, spinal manipulative therapy broadly defined includes all procedures where hands are used to mobilize, adjust, manipulate, apply traction, massage, stimulate, or otherwise influence the spine or the paraspinal tissues with the aim of influencing the patient's health [4] These results were confirmed by Parkin-Smith in his study on the efficacy of manipulation in the treatment of mechanical neck pain, where an increase in cervical range of motion and a decrease in neck pain were also recorded [5].

The first rib is commonly fixated in flexion, when the rib is palpated for motion it appears elevated and does not recede from the palpating finger. The restriction of movement is common following hyperextension injuries of the cervical spine, due to the attachments of the cervical spine [6]. As a result of the first ribs involvement in cervical spine path mechanics, it has been noted by Haley [7] that first rib dysfunctions are under diagnosed or misdiagnosed due to a lack of assessment or focus of assessment. However, it has been stated that a first rib dysfunction is one of the most common and over-looked dysarthrodial conditions [8]. According to Douglas ,first rib manipulation had a positive immediate and short-term effect on the following ranges of motion of the cervical spine, flexion, extension, right lateral flexion and left lateral flexion [9]. The adjustment of the first rib caused no immediate or short-term relief from pain itself, and so is not an effective form of treatment for patients suffering from mechanical neck pain if used in isolation [9]. This is supported by Johnson (1995:134)who found that the early detection and treatment of a first rib dysfunction often leads to significant and lasting relief from the condition for the patient [10]. Therefore, this research aims to investigate the effectiveness of first rib manipulation as an adjunct to the treatment of neck pain.

\section{Active Exercises}

\section{Stretching Exercises and Strengthening Exercises}

It involves the static contraction of a muscle without any visible movement in the angle of the joint. In simple terms this means that in these exercises the length of the muscle does not change. As it is continued for the cervical spine it will gain strength in neck [11]. Jonathan Bean [10] suggest that performing specific muscle-strengthening exercises may be a helpful strategy for many women with chronic neck pain. (The researchers have investigated the effectiveness of each exercise with electromyography, which measures muscle-generated electrical activity).

\section{Materials and Methods}

This is an observational, correlation study design. The study was done in Baqai Institute of Diabetology \& Endocrinology, where working and non-working female patients were seen more coming with neck pain in physiotherapy department and were selected randomly. They were also informed, and permission was taken from the female patients before starting the research. The sample size consisted of thirty-four female patients, which included working and non-working middle age and old age women.

Thirty-four female patients with a diagnosis of neck pain with acute and chronic conditions were required for the study were obtained by the Baqai Institute of Diabetology \& Endocrinology. It was decided that all the patients will be manipulated in supine position according to standardize treatment procedure. Only participants between the ages of 30 to 60 years of age were included into the study, Women working and non-working women with complaint of neck pain with or without upper extremity symptoms T1 Syndrome, cervical myofascial pain, cervical disco-genic pain, cervical facet syndrome, cervical spondylosis, Cervical strain and sprain, acute Whiplash Injury, degenerative changes in cervical spine, Headache, Cervical Muscle spasm, Cervical Joint stiffness, Fibromyalgia. Patients were only accepted if they had read and signed the informed consent form, undergone a full history and cervical examination. Patients were requested to participate in the entire protocol in order to avoid exclusion.

Any persons that at the time were currently taking either anti-inflammatory or analgesic medication were required to cease the intake 48 hours prior to consultation. Participants with any contra-indications to manipulation were not included into the study $[12,13]$. If any major lifestyle changes (e.g. rigorous exercise) were made while involved with the study, that participant was excluded from the study. Females, pregnant or lactating, at the time of the study, were excluded. Patients were excluded from the study if the allotted number of consultations or the clinical protocol were not adhered to patients including children and men were excluded from the study. Patients including congenital deformity were excluded from study. Patients with burn were excluded from study. Diagnosis was made on the basis of following test. Distraction test foraminal compression test, muscle length test for neck muscles.

\section{Research Methodology/Procedure}

In order to ensure that the patient's completely understood the research and to protect their interests, the patients were 
required to read and sign the Consent form and were asked to fill a Questionnaire with closed ended questions (Appendix A) Female Patients both working (included dentist, nurse, teacher, housemaid, computer operator, car driving trainer and bank employee) and non-working (house-wife), diagnose with acute or chronic neck pain that fulfill the inclusion and exclusion criteria were included in this study. Assessment of the patient was done with spurling's test, distraction test, Muscle length test of trapezius, sternocleidomastoid, Levator scapulae and scalene (anterior, posterior, medius) (Appendix B) were performed and a closed ended questionnaire filled, pain intensity by numeric pain rating scale (NRS) and functional ability was assessed by Vernon Neck Disability Index (NDI) questionnaire (Appendix A). Then the patient was treated by 1st rib manipulation depression (Appendix C) followed by active exercises which included stretching and strengthening exercises (Appendix D). After the initial consultation, patients were required to attend 2-week follow-up which required 6 days of physical therapy visits in a week in which first rib depression manipulation was given 3 sessions in a week followed by 3 sessions of active exercise program which included stretching and strengthening exercises.

\section{Measurements}

To measure the subjective outcomes, the patients were required to complete the Vernon Neck Disability Index (NDI) form (Appendix A) and the NRS Pain Rating Scale in the questionnaire form.

\section{Data Analysis}

Data was analyzed using SPSS. Simple analytical method was used with the help of graph.

\section{Result}

The graphical representation shows that among 34 patients the incidence of neck pain seen in 21 middle age patients was found to be more as compared to 13 old age women patients. (Graph 1) The graphical representation shows that among 34 patients, working women 18 were seen more effected with neck pain as compared to housewife 16. (Graph 2). The graphical representation shows that among 34 patients, 21 female patients were seen with radiating neck pain, which were found more commonly present in both middle age and old age women as compared to non- radiating pain present (Graph 3). The graphical representation shows that among 34 patients during the Muscle Length test was performed; most commonly affected muscle seen was trapezius muscle which was seen as mostly affected during neck pain (Graph 4). The graphical representation shows that among 34 patients most commonly neck pain in women were seen present in acute conditions (15 middle age $\&$ old age) rather than chronic conditions ( 6 middle age $\& 8$ old age) (Graph 5). The graphical representation shows that among 34 patients, effectiveness of treatment was seen in middle age patients as compared to old age patients. 20 patients were improved with effective treatment, 12 patients were seen with slight recovery and 2 patients were not at all got any effective treatment (Graph 6).
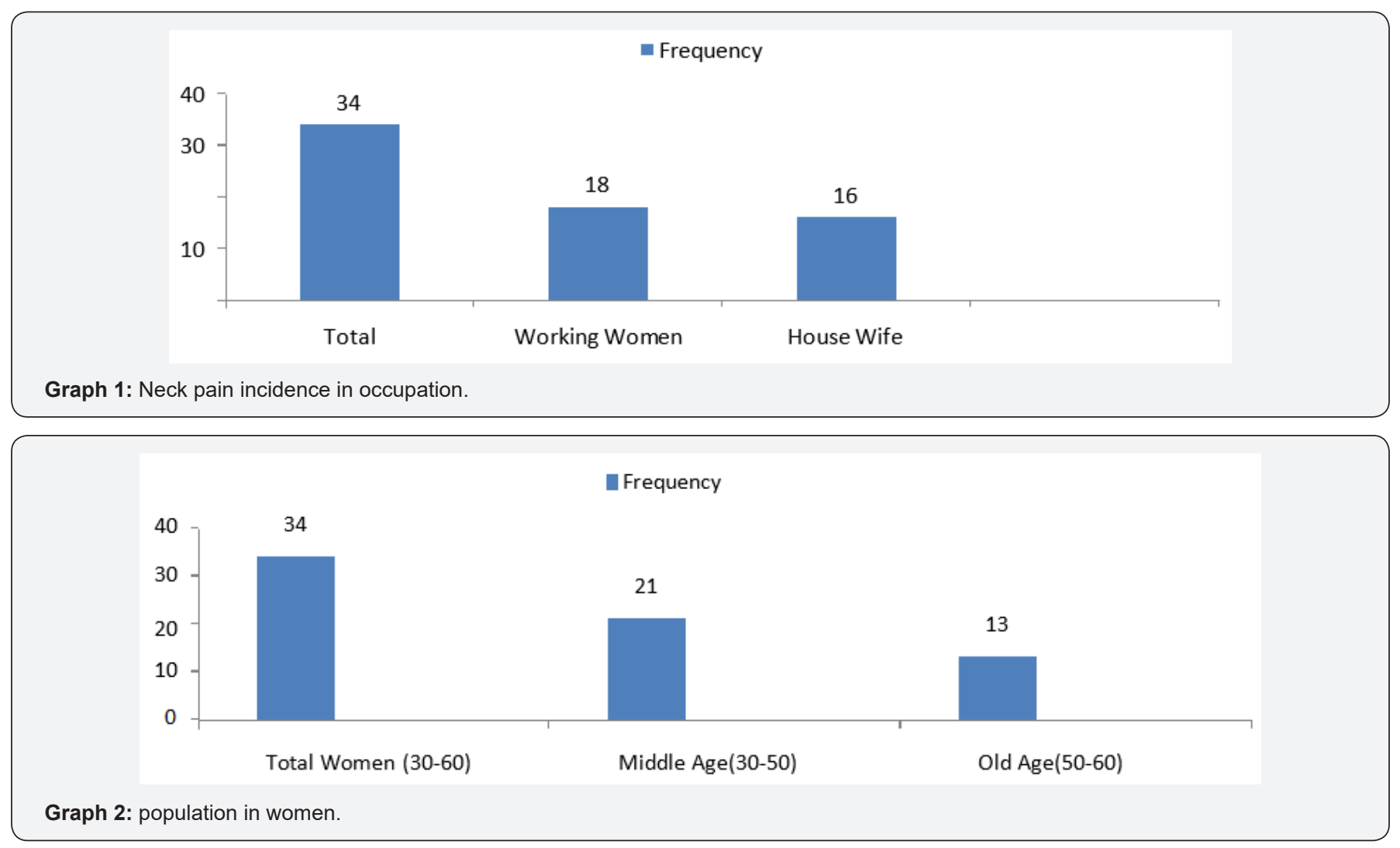

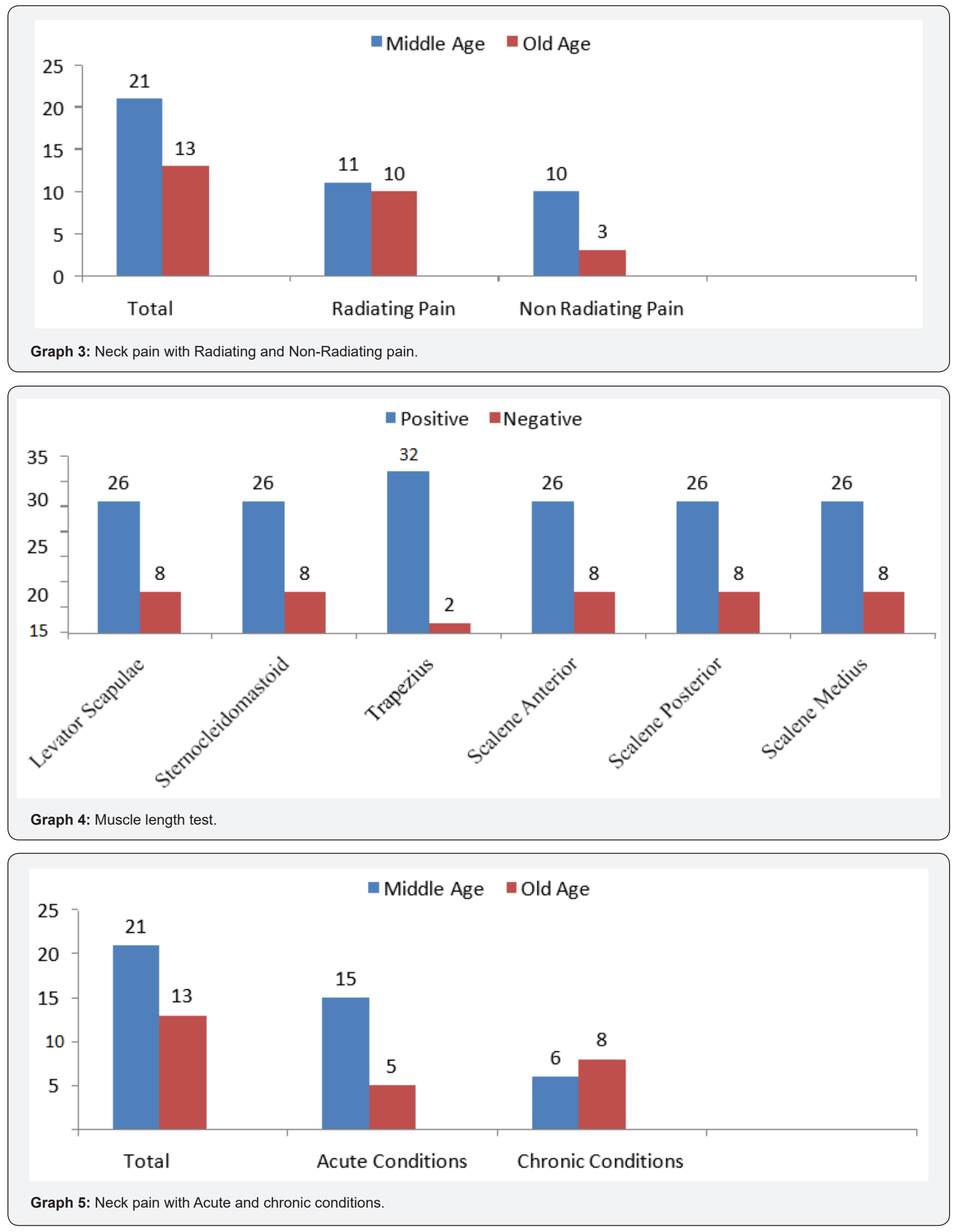


\section{aiddle Age $\quad$ Old Age}

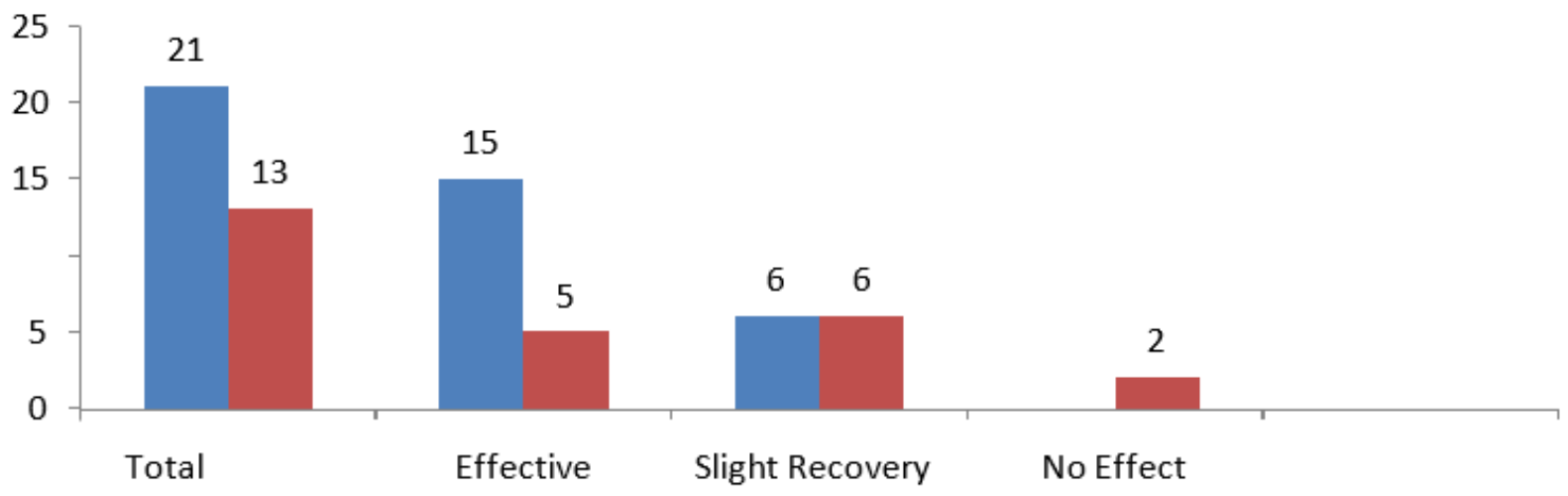

Graph 6: Effectiveness of treatment.

\section{Discussion}

A study suggested that this finding may strengthen the assumption that there is an association between working conditions and the occurrence of neck and upper extremity complaints [14]. Alternatively, chronic disease and other ailments may be more important in older people and be the main reason for encountering them in general practice. Again, additional (longitudinal) research is needed to establish the association between work, age, and the occurrence of neck and upper extremity complaints. Colin Douglas Brown study researched on the effectiveness of first rib adjustment as an adjunct to the treatment of mechanical neck pain and suggested that there was limited evidence for any difference in treatment effects between the three treatment groups [15]. Only CMCC score showed significant treatment differences between the first rib group and cervical spine group (first rib was better) and between the cervical spine group and first rib plus cervical spine group (first rib plus cervical spine group was better).

Thus, it appeared that first rib manipulation was more beneficial for CMCC score than cervical spine manipulation, in terms of patients' perception. In comparison with the placebo treatment, there were many significant treatment effects. Left lateral flexion was improved in the first rib group and the first rib plus cervical spine group compared with the placebo. Extension was improved in the cervical spine group compared with placebo. CMCC was improved in the first rib group relative to placebo, and NRS was significantly improved in all treatment groups compared with placebo. Where the $\mathrm{p}$ value was not significant, trends were demonstrated. Thus, any treatment was better than placebo for most outcomes. This is evident that $1 \mathrm{st}$ rib manipulation has an evidence based successful treatment for neck pain, but different research suggest different techniques of treatment. Miller et al. [16] suggested that, if manual therapy, including manipulation or mobilization, combined with exercise improves pain, function/disability, quality of life, global perceived effect, and patient satisfaction for adults with neck pain with or without cervicogenic headache or radiculopathy.

Kay TM [17] suggested with a background study that Neck disorders are common, disabling and costly. The effectiveness of exercise as a physiotherapy intervention remains unclear and came with results that Moderate quality evidence demonstrates patients are very satisfied with their care when treated with therapeutic exercise. Low quality evidence shows exercise is of benefit for pain in the short term and for function up to long-term follow-up for chronic neck pain. After the 1st rib manipulation patients were instructed in a stretching and strengthening program. Recent guidelines and reviews have supported the use of exercise to decrease pain, improve function and reduce disability in a patient population with neck pain [18].

\section{Limitations of the Study}

In Baqai Institute of Diabetology \& Endocrinology where the research is being performed does not have any heating modalities, thera band, dumbbell's, mechanical traction and exercise ball, that's why designing in treatment techniques were limited so it was unable to select use of any equipment to achieve any improvement or reduction in pain which is realized at present time. A new approach is needed to solve the problem and to progress towards the designing of better-quality clinical practice guidelines.

\section{Recommendations}

Measurements of range of motion over the cervical spine region should have been taken before and after treatment for better results. This could have had an effect on the eventual outcome of the results. Further research could be done in order to compare the treatment given in seated, prone and supine positions. Future efforts should focus on the study of noninvasive interventions for patients with radicular symptoms and on the design and evaluation of neck pain prevention strategies. Further research can also be done on males and the effectiveness 
of treatment in males. Further research should be done with a home exercise program and prevention tips should also been given to the patient to reduce chances of increase in neck pain so that the patient find less difficulty in their work and leisure activities.

\section{Conclusion}

In this study, from a group of 34 female patients, 20 (14 acute $\& 6$ chronic conditions which included 15 middle age and 5 old age women) patients received effective treatment and had NDI score less than $14 \%$, with reduce sign and symptoms and improved strength of cervical muscles with greater functional ability so they were send back to their work while 12 (12 chronic condition which included 6 old age and 6 middle age woman) patients did not receive more effective treatment as their NDI score was more than $15 \%$ with aggravating sign and symptoms and reduced strength of cervical muscles with lesser functional ability. 2 (chronic old age) patients did not receive any improvement after the course of 2-week treatment sessions. Treatment were seen to be effective but mostly in acute patients, so it is suggested a new approach is needed to solve the problem for chronic patients and to progress towards the designing of better-quality clinical practice guidelines, long duration of treatment, its techniques and better quality of exercises.

\section{References}

1. Blyth FM, Hoy DG, March LM (2011) Musculoskeletal pain on the global stage: what next? Translational behavioral medicine 2(1): 117-119.

2. Goldin PR, Gross JJ (2010) Effects of mindfulness-based stress reduction (MBSR) on emotion regulation in social anxiety disorder. Emotion 10(1): 83-91.

3. Vincent C (2012) Parenting: Responsibilities, Risks and Respect. Institute of Education, University of London: London.

4. Douglas BS An investigation into the efficacy of a first rib manipulation in individuals experiencing mechanical neck pain: a pilot study (Doctoral dissertation).

5. Parkin-Smith GF The efficacy of spinal manipulative therapy in the treatment of mechanical neck pain (Doctoral dissertation).

6. Brown CD. The effectiveness of first rib adjustment as an adjunct to the treatment of mechanical neck pain (Doctoral dissertation).
7. Haley RW, Kurt TL, Hom J (1977) Is there a Gulf war syndrome? searching for syndromes by factor analysis of symptoms. Jama 277(3): 215-222.

8. Grassi R, Rotondo A, Catalano O, Salzano A, Parisi B, Amitrano M, Fanucci A (1994) La videoproctografia (VPG) nello studio del prolassomucosorettale e dellaintussuscezionerettale. Annalitaliani di Chirurgia 65(2): 167-170.

9. Gentleman RC, Carey VJ, Bates DM, Bolstad B, Dettling M, et al. (2004) Bioconductor: open software development for computational biology and bioinformatics. Genome biology 5(10): R80.

10. Schulte PA, Burnett CA, Boeniger MF, Johnson J (1996) Neurodegenerative diseases: occupational occurrence and potential risk factors, 1982 through 1991. American journal of public health 86(9): 1281-1288.

11. Verkhoshanskiĭ IV (2006) Special strength training: A practical manual for coaches. Ultimate Athletic Concepts.

12. Mayson DJ, Kiely DK, LaRose SI, Bean JF (2008) Leg Strength or Velocity of Movement Which Is More Influential on the Balance of Mobility Limited Elders? American journal of physical medicine \& rehabilitation/Association of Academic Physiatrists 87(12): 969-976.

13. Douglas BS An investigation into the efficacy of a first rib manipulation in individuals experiencing mechanical neck pain: a pilot study (Doctoral dissertation).

14. Bot SD, Terwee CB, van der Windt DA, Bouter LM, Dekker J, et al. (2004) Clinimetric evaluation of shoulder disability questionnaires: a systematic review of the literature. Annals of the rheumatic diseases 63(4): 335-341.

15. Travis LB, Rabkin CS, Brown LM, Allan JM, Alter BP, et al. (2006) Cancer survivorship-genetic susceptibility and second primary cancers: research strategies and recommendations. Journal of the National Cancer Institute 98(1): 15-25.

16. Lozano R, Naghavi M, Foreman K, Lim S, Shibuya K, et al. (2012) Global and regional mortality from 235 causes of death for 20 age groups in 1990 and 2010: a systematic analysis for the Global Burden of Disease Study 2010. The lancet 380(9859): 2095-2128.

17. Porrata LF, Ristow K, Colgan JP, Habermann TM, Witzig TE, et al. (2012) Peripheral blood lymphocyte/monocyte ratio at diagnosis and survival in classical Hodgkin's lymphoma. Haematologica 97(2): 262-269.

18. University R (2009) Evaluation of outcomes in patients with neck pain treated with thoracic spine manipulation and exercise: a case series. NZ Journal of Physiotherapy 37(2).

\section{Your next submission with Juniper Publishers}

\section{will reach you the below assets}

- Quality Editorial service

- Swift Peer Review

- Reprints availability

- E-prints Service

- Manuscript Podcast for convenient understanding

- Global attainment for your research

- Manuscript accessibility in different formats

( Pdf, E-pub, Full Text, Audio)

- Unceasing customer service

Track the below URL for one-step submission

https://juniperpublishers.com/online-submission.php 